\title{
Stress impacts the fidelity but not strength of emotional memories
}

\author{
Maheen Shermohammed $^{\mathrm{a}}$, Juliet Y. Davidow ${ }^{\mathrm{a}}$, Leah H. Somerville ${ }^{\mathrm{a}}$, and Vishnu P. Murty ${ }^{\mathrm{b}}$ \\ aDepartment of Psychology and Center for Brain Science, Harvard University, Cambridge, MA \\ 02138, USA

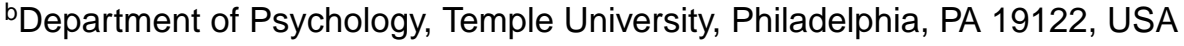

\begin{abstract}
Psychological stress during memory encoding influences resulting memory representations. However, open questions remain regarding how stress interacts with emotional memory. This interaction has mainly been studied by characterizing the correct identification of previously observed material (memory "hits"), with few studies investigating how stress influences the endorsement of unobserved material as remembered (memory "false alarms"). While hits can provide information about the presence or strength of a memory representation, false alarms provide insight into memory fidelity, indicating to what extent stored memories are confused with similar information presented at retrieval. This study examined the effects of stress on long-term memory for negative and neutral images, considering the separate contributions of hits and false alarms. Participants viewed images after repeated exposure to either a stress or a control manipulation. Stress impaired memory performance for negative pictures and enhanced memory performance for neutral pictures. These effects were driven by false alarms rather than hits: stressed participants false alarmed more often for negative and less often for neutral images. These data suggest that stress undermines the benefits of emotion on memory by changing individuals' susceptibility towards false alarms, and highlight the need to consider memory strength and fidelity to characterize differences in memory performance.
\end{abstract}

\section{Introduction}

The long-term encoding of memories is modulated by stress. Imagine a stressful situation, such as witnessing a crime. Would memory of the criminal be better or worse than if the same event were observed in a less stressful circumstance, like behind the safety of a television screen? A long-standing belief is that increases in stress at encoding enhance subsequent memory (Cahill \& McGaugh, 1998; LaBar \& Cabeza, 2006). In support of this theory, several studies have demonstrated improvements in memory performance when

\footnotetext{
Address correspondence to: Vishnu P. Murty, Department of Psychology, Temple University, Weiss Hall, 1701 N 13th St, Philadelphia, PA 19122, vishnu.murty@temple.edu (V. P. M.).mshermohammed@g.harvard.edu (M. S.), jdavidow@ fas.harvard.edu (J. Y. D.), somerville@fas.harvard.edu (L. H. S.).

Publisher's Disclaimer: This is a PDF file of an unedited manuscript that has been accepted for publication. As a service to our customers we are providing this early version of the manuscript. The manuscript will undergo copyediting, typesetting, and review of the resulting proof before it is published in its final citable form. Please note that during the production process errors may be discovered which could affect the content, and all legal disclaimers that apply to the journal pertain.
} 
encoding is preceded by or concomitant with a psychological stress induction (Henckens, Hermans, Pu, Joëls, \& Fernández, 2009; Hoscheidt, LaBar, Ryan, Jacobs, \& Nadel, 2014) or pharmacological administration of the stress response hormone cortisol (Abercrombie, Kalin, Thurow, Rosenkranz, \& Davidson, 2003; Buchanan \& Lovallo, 2001).

Nonetheless, a deeper examination of the current research suggests that the relationship between stress and memory is complex. Although stress has been shown to enhance memory in some circumstances, it has also been found to impair or have no effect on memory in others (de Quervain, Roozendaal, Nitsch, McGaugh, \& Hock, 2000; Maheu, Collicutt, Kornik, Moszkowski, \& Lupien, 2005; Payne et al., 2006; Schwabe \& Wolf, 2010; Smeets, Otgaar, Candel, \& Wolf, 2008). For example, Schwabe \& Wolf (2010) used the socially evaluated cold pressor test to induce an acute psychological and physiological stress response while participants encoded words, and found that participants under stress performed worse on free recall and recognition tests 24 hours later. Meanwhile, other studies found that increasing cortisol levels during the encoding of word lists revealed no effects on recall 24 hours later (de Quervain et al., 2000; Smeets et al., 2008).

This complexity is further magnified by the introduction of emotional valence. On its own, negative emotional valence typically enhances memory (Hamann, 2001; Yonelinas \& Ritchey, 2015), in part through its association with arousal (Cahill, Prins, Weber, \& McGaugh, 1994; Rimmele, Lackovic, Tobe, Leventhal, \& Phelps, 2016). When both negative and neutral stimuli are encoded under acute stress, some studies show valencespecific enhancements (Buchanan \& Lovallo, 2001; Cornelisse, van Stegeren, \& Joëls, 2011), impairments (Maheu et al., 2005; Payne et al., 2006; Smeets, Jelicic, \& Merckelbach, 2006), both enhancements and impairments (Jelici, Geraerts, Merckelbach, \& Guerrieri, 2004; Payne et al., 2007), and still others show no difference between negative and neutral material (Abercrombie et al., 2003; Henckens et al., 2009; Schwabe \& Wolf, 2010). Moreover, meta-analytic evidence shows that valence does not reliably moderate stressmemory encoding interactions (Shields, Sazma, McCullough, \& Yonelinas, 2017), and highlights the need to better characterize how and when stress and valence interact to modulate long-term memory.

Most of the previous research on stress and memory characterize how stress during encoding influences later recall or recognition, and thus focus on whether individuals remember memoranda (i.e., can endorse a previously experienced event as being old). However, another critical component of memory success may be characterizing the fidelity of memory. That is, a correctly recalled memory can be qualified by an individual's ability to discriminate that memory from competing information with similar properties (i.e., similar new information that has not been previously observed). One way to examine both memory presence and fidelity is to separately assess "hits", when someone accurately identifies previously encoded information, and "false alarms", when a new stimulus is incorrectly identified as old. It is possible that factors like stress and valence interact to influence these components of memory performance differently, contributing towards the difficulty in reconciling findings across studies, which typically combine them into one overall measure of memory performance. Examining memory presence and fidelity separately may provide a more comprehensive psychological picture, de-conflating two qualitatively different aspects 
of memory performance. This may be particularly relevant for memory under stress, as the neurocognitive systems underlying stress have been shown to influence memory fidelity in other behavioral contexts (Murty \& Adcock, 2017; Yonelinas \& Ritchey, 2015).

In the present study, we examined the effects of stress on encoding negative and neutral images, separately considering the contributions of hits and false alarms to memory performance. The data analyzed in this study were originally collected as part of a larger neuroimaging study (Shermohammed et al., 2017), however the current manuscript focuses on the behavioral data because the sample size is underpowered to conduct subsequent memory analyses on the neuroimaging data. In addition, although this previous work investigated the effects of stress on emotion regulation, the planned analyses in the present work focus on the behavioral data from the passive viewing conditions. During this study, participants observed images after experiencing either an acute stress induction, using an adaptation of the Trier Social Stress Test (Kirschbaum, Pirke, \& Hellhammer, 1993), or a control manipulation. Here, we analyze behavioral data collected from a surprise memory test approximately two weeks after encountering the pictures. Analyses first validated the effectiveness of the stress induction and then examined the effects of stress on recognition memory performance for passively viewed negative and neutral pictures. To better resolve how retrieval-related processes may influence these effects, we analyzed the relative contributions of hits and false alarms to overall memory. Finally, we assessed individual differences in the effects of stress on memory.

\section{Method}

\subsection{Participants}

Fifty-six young adults aged 18-23 participated in this study. Two participants were excluded from analyses due to non-compliance. In addition, since the purpose of the present study was to examine the effects of stress resulting from a stress manipulation, 2 participants in the control group that reported increases in stress and 5 participants in the stress group that did not report increases in stress were excluded (see section 2.6.1 below), leaving a final sample of 47 ( 23 females; 23 controls; mean age $21.13+/-1.67$ years). In the current study, we operationalize stress as the psychological perception of stress. We chose to exclude participants based on self-report rather than a physiological measure like cortisol because not all situations perceived as stressful result in a cortisol response (Hellhammer, Wüst, \& Kudielka, 2009), and cortisol increases are not uniquely provoked by events perceived as stressful (Anisman \& Merali, 1999). Furthermore, the use of cortisol as an objective index of stress may introduce gender bias, as cortisol is more reliably and robustly elicited in males than females (Childs, Dlugos, \& De Wit, 2010; Kirschbaum, Kudielka, Gaab, Schommer, \& Hellhammer, 1999; Kirschbaum, Wust, \& Hellhammer, 1992), including in the present study (see section 3.1 below).

All participants were right-handed, non-smokers, proficient in English, and not currently receiving treatment for psychological or neurological disorders. In an effort to recruit a more representative sample, no more than $25 \%$ of participants were current or former students at Harvard University. All participants provided informed written consent. Research 
procedures were approved by the Committee on the Use of Human Subjects at Harvard University and by the Army Human Research Protections Office (AHRPO).

\subsection{Incidental Encoding Task}

Participants viewed 48 negative and 24 neutral images from the International Affective Picture System (IAPS) database (Lang, Bradley, \& Cuthbert, 1997), all of which included depictions of people and were presented in color. Two sets of negative images were constructed with the same average normative valence (means $=2.762$ and 2.768; range $=$ 1.79-3.9) and arousal (means $=5.45$ and 5.34; range $=3.85-6.99$ ) on a scale of $1-9$ (Lang et al., 1997). Neutral images had neutral normative valence (mean $=5.078$; range $=4.42-5.59$ ) and lower arousal (mean $=3.43$; range $=2.43-4.16$ ) ratings on the same scale.

While viewing the negative and neutral images, participants were instructed to decrease their emotional response to half of the negative images using cognitive reappraisal, an emotion regulation strategy (see Shermohammed et al., 2017), and instructed to passively view the other half of the negative images and all of the neutral images. This protocol has frequently been employed in prior work to examine emotion regulatory processes (Ochsner et al., 2004), but is nonetheless sensitive to differences in subjective affective experience by valence. We limit current analyses to the look-neutral and look-negative conditions to avoid confounding effects of emotion regulation, such as possible deeper encoding. Further, in the prior study stress did not systematically influence emotion regulation, thus we did not have strong theoretically driven hypotheses to investigate a moderating role of emotion regulation. We have included the memory results for the decrease-negative condition in the Supplemental Figure 1.

For each trial of the task, a 2-sec instructional cue first informed participants whether they were to passively view ("LOOK") or reappraise ("DECREASE") an image that followed. The image was displayed $(8 \mathrm{sec})$, and then participants provided self-reported negative affect ratings on a scale of $1-5$ using a button response box held in the right hand $(3 \mathrm{sec})$. Jittered fixation periods were presented between the image display and the rating scale $(1-5 \mathrm{sec})$ and between the rating scale and the next trial $(2-8 \mathrm{sec})$. Pictures were presented over $3 \mathrm{fMRI}$ scanning runs with 24 trials each, and run order was counterbalanced across participants. This was an implicit encoding task; participants were not aware that their memory would be tested on these pictures.

\subsection{Acute Stress Manipulation}

The goal of the experimental session was to elicit a stress response that would persist throughout fMRI scanning. To achieve this, we administered two stress induction tasks, which are described in greater detail in (Shermohammed et al., 2017) and summarized briefly here.

The Speech Stress Induction Task was adapted from the preparation phase of the Trier Social Stress Test (Kirschbaum et al., 1993). Before the MRI scan, participants in the stress group were told that immediately after the scan they would be giving a speech to a panel of judges that would be assessing their verbal and nonverbal behavior. Participants in the control group were instead told that they would write a story after the MRI scan. Both groups were given 3 
min to prepare for their respective task. Over the course of the session, participants were reminded of their forthcoming speech (stress group) or story-writing (control group) task, which they were later informed they would not have to complete.

The Math Stress Induction Task was adapted from the Montreal Imaging Stress Task (Dedovic et al., 2005). During fMRI scanning, participants answered math problems by selecting a number on a rotary dial. Participants in the stress group received more complex math problems and a very limited amount of time to answer them. The time limit changed adaptively to ensure poor performance, and was indicated by a progress bar and a tone of escalating frequency. Participants in the stress condition were also given negative social evaluative feedback about their task performance by the experimenter. They were told that the average performance was quite high, and during the task were given a false indication that their performance was substantially worse than their peers. They were also told that they needed to get at least $70 \%$ of problems correct for their data to be usable, and after every run of the task, stressed participants were given feedback from the researcher expressing surprise and disappointment at their poor performance. Control participants completed a similar version of the task that taxed mathematical processes but did not include the potentially stressful components. The control version of the task was self-paced (hence, no time pressure), participants did not receive the social evaluative feedback, and the mathematical operations were easier.

In each of three runs of the Math Stress Induction Task, participants completed a 2-min block of math, which was preceded and succeeded by $30 \mathrm{sec}$ of resting baseline where the visual interface was displayed without a math problem. Heart rate was continuously monitored during both the math and rest blocks, serving as a measure of physiological stress in response to this task.

\subsection{Memory Retrieval Task}

The surprise retrieval task occurred approximately 2 weeks after the encoding study visit (mean delay $=14.9$ days $+/-2$ days; range $=13-21$ days; see Supplemental Figure S2 for more information on the distribution of this delay). Participants completed the task on their computers at home via the Qualtrics (Provo, UT) software. While participants knew they would be completing some tasks at home, they were not aware until the instructions were given that their memory would be tested. Instructions for the task were administered verbally over the phone and participants were given the opportunity to complete a practice trial and ask questions. Participants were requested to complete the task in a quiet room and in one sitting immediately after getting off the phone. Previous research suggests that cognitive tasks completed in remote environments are comparable with laboratory tasks (Crump, Mcdonnell, \& Gureckis, 2013; Murty, FeldmanHall, Hunter, Phelps, \& Davachi, 2016). Although we have no reason to believe participants were not compliant, we have no objective means to verify they complied with instructions exactly as given.

During each trial of the task, participants were presented with a black-and-white image. They indicated whether this image was "Old", as in they had seen it during the encoding task at their initial study visit, or "New", with a button press. Next, participants were asked to indicate how confident they were in this response on a scale from 1 ("Not Sure") to 4 
("Very Sure). Finally, for the pictures that were indicated as "Old", a third question was asked probing what the instruction was for the picture during the encoding task; participants could select "LOOK", "DECREASE", or "I don't know". This question was used as an assessment of source memory for pictures that were hits; for pictures that were false alarms, because there can be no source memory for unobserved images, this measure can only assess how vivid the inaccurate memory for the item is.

In total, 144 IAPS images were presented, including the 72 images from the encoding task and 72 new foils ( 48 negative and 24 neutral). Negative foils were equated to encoding images in normative valence $($ mean $=2.768$; range $=1.77-3.89)$ and arousal $($ mean $=5.56$; range $=3.95-7.29)$ ratings.

\subsection{Study Procedure}

After consenting, participants were introduced to the tasks and given the opportunity to practice them. For the math task, all participants practiced the untimed, easier version. Next, to give participants time to reduce any initial stress associated with entering the laboratory environment, participants watched the first 20 min of an episode from the Planet Earth TV series titled "Ocean Deep," a mildly positive, informative video about animals in the ocean (Denson, Mehta, \& Ho Tan, 2013). Participants then received instructions about the speech (stress group) or story (control group) task and completed the 3-min preparation phase where they could outline their speech or story.

Next, participants completed three runs each of the math and encoding tasks in alternating order, with math always being first. Each run of the encoding task contained a different set of images, such that every image was observed once during encoding. After all task runs, participants were asked if they knew what was happening next to give them an opportunity to volunteer any suspicions about the deceptive nature of the speech task. During the full debriefing, we asked participants whether they found the speech/story and math tasks stressful, which gave them another chance to volunteer any suspicions they had. Finally, participants completed follow-up questionnaires and assessments and provided mood ratings for the fifth time. They were then compensated for their time and thanked for their participation. Approximately 2 weeks later, participants completed the memory retrieval task at home.

\subsection{Stress Response Indices}

To evaluate whether stress was in fact induced in the stress group, we monitored participants' self-reported stress, heart rate, and cortisol levels. We compared the stress and control groups along each of these indices using Welch's unequal variances t-test, which employs an adjustment to the degrees of freedom to remove assumptions about equal variances between groups. For more detailed Group by Time interactions, see (Shermohammed et al., 2017).

2.6.1. Self Report-To assess subjective stress experience, five times over the course of the study participants reported their subjective stress levels by rating to what extent they felt "stressed" right now on a scale of 1-5 (Very slightly / not at all, A little, Moderately, Quite a 
bit, Extremely). This occurred immediately after the Planet Earth video, after each run of the encoding task, and after debriefing. Each participant's overall self-reported stress level was computed as the average of the 3 "stressed" ratings provided after each encoding run, corrected for the initial baseline rating. We used this average self-reported stress level to exclude any "non-responders" in the stress group (those who never rated their stress levels as higher than baseline, $\mathrm{n}=5$ ) and any participants in the control group who were stressed (exhibiting an average stress rating increase from baseline was greater than 0.67 , which was 1.5 times the interquartile range above the upper quartile, $\mathrm{n}=2$ ).

2.6.2. Salivary Cortisol—Participants provided salivary cortisol samples immediately after the Planet Earth video and after the second and third runs of the encoding task. Sampling commenced after the second rather than first run to account for the delay to peak in cortisol levels evoked by stress (Dickerson \& Kemeny, 2004). Samples were collected by placing Saliva-Bio oral swabs under the tongue for 3-4 min. They were then immediately stored in Swab Storage Tubes (Salimetrics, Inc., State College, PA) at $-20^{\circ} \mathrm{C}$. To maximize the integrity of the samples, participants were instructed to refrain from exercising for $12 \mathrm{hr}$, consuming caffeine for $2 \mathrm{hr}$, eating or drinking anything but water for $30 \mathrm{~min}$, and brushing their teeth for $1 \mathrm{hr}$ before the experiment. To reduce the effects of diurnal variation, all MRI sessions were scheduled between 12:30 and 5:30 p.m. Once data collection was complete, samples were shipped frozen overnight to the Kirschbaum laboratory (Technische Universit t, Dresden, Germany) for analysis with chemiluminescence immunoassay kits (IBL International, Hamburg, Germany). Samples were analyzed in singlets, and the interassay coefficient of variation was less than $8 \%$. To evaluate each participant's evoked cortisol level, we used the formula for area under the curve with respect to the increase (Pruessner, Kirschbaum, Meinlschmid, \& Hellhammer, 2003), a measure that corrects for the pre-task baseline level.

2.6.3. Heart Rate-Heart rate was monitored with a fiber-optic oximetry sensor on the left ring finger using BIOPAC Systems MP150 and OXY-MRI (Nonin Medical, Inc., Plymouth, MN) hardware and AcqKnowledge software. Technical difficulties with the AcqKnowledge software resulted in full data loss for $n=6$. Any participant with at least one full run of heart rate data was included in these analyses, resulting in $n=41$ usable data sets. Heart rate acquired during each run of the math task was compared with the resting baseline preceding it to provide an indicator of each participant's autonomic stress responsiveness.

\subsection{Statistical Analyses}

To evaluate the effects of stress and valence on memory performance, we conducted twofactor ANOVAs using group (stress, control) as a between-subjects factor and valence (negative, neutral) as a within-subjects factor. The primary measure of memory performance was d-prime, a statistic from signal detection theory that is obtained by taking the difference between the z-transformed hit rate and false alarm rate (Macmillan \& Creelman, 2005). This index is preferred to hit rate alone, as it is sensitive to response criteria and bias (Rotello \& Macmillan, 2007). We conducted follow-up analyses examining the effects of group and valence on hit rate and false alarm rate separately in order to understand the driving mechanism behind any d-prime differences. 
We conducted additional follow-up analyses on the false alarms, which showed significant group by valence interactions. These analyses examined the consistency of stress-related differences in false alarms at the individual subject level. We used each of the subject-level stress response indices (self report, cortisol, and heart rate) to perform 3 two-way repeatedmeasures ANOVAs examining the effects of stress and valence on false alarm rate. Reported statistical results were corrected for 3 comparisons by controlling for the false discovery rate (FDR) using the Benjamini-Hochberg method. We also conducted a follow-up analysis to assess whether group-valence interactions were moderated by confidence. To do so, average confidence level reported in each condition was input into a repeated-measures ANOVA examining the effects of group, valence, and confidence on false alarm rate.

Finally, we used source memory responses (i.e., participants' memory of what the instruction was for the picture at encoding: either making a source attribution of "Look" or "Decrease", or indicating "I don't know") to conduct exploratory follow-up analyses on false alarm results. Specifically, we examined whether source attributions were made for images to which participants had false alarmed. To do so, we conducted a trial-by-trial mixed-effects logistic regression to assess how group and valence predicted the likelihood of making a source attribution, with a binary outcome variable of 1 corresponding to making a source attribution and 0 corresponding to indicating "I don't know." Note that since this was an analysis of false alarms, the images had not actually been previously observed, so any source judgment would be inaccurate; we simply assess here whether they chose to make this incorrect judgment or to refrain from making it. To control for the within-subject nature of the data, we included a random effect of participant. All statistical analyses were performed in R 3.2.3 (R Core Team, 2015).

\section{Results}

\subsection{Stress Manipulation Check}

The success of the stress induction was assessed by comparing stress and control groups on self-reported stress levels, changes in heart rate in the math task vs. rest, and evoked cortisol levels. As previously reported (Shermohammed et al., 2017), we found greater self-reported stress $(\mathrm{t}(46.695)=4.106, \mathrm{p}<.001)$, greater math-induced elevation in heart rate $(\mathrm{t}(32.777)=$ $6.032, \mathrm{p}<.001)$, and greater evoked cortisol levels $(\mathrm{t}(42.414)=2.22, \mathrm{p}=.032)$ in the stress versus control group.

Comprehensive analyses examining changes in stress measures relative to baseline and over time using two-factor ANOVAs are reported in more detail elsewhere (Shermohammed et al., 2017). Briefly: there was a significant group $\times$ sample number interaction for selfreported stress ratings $(\mathrm{F}(4,50)=12.89, \mathrm{p}<.001)$, such that ratings were elevated for the stress group after baseline and before debriefing. Heart rate levels increased during the math task relative to the baseline period preceding it for only the stress group $(F(1,46)=37.52$, $p$ $<.001)$. Finally, there was a significant group $\mathrm{x}$ sample number interaction for cortisol levels $(\mathrm{F}(2,101)=3.880, \mathrm{p}=.024)$, with no difference at baseline and an increase for the stress group at the subsequent two samples. Consistent with previous work (Childs et al., 2010; Kirschbaum et al., 1999, 1992), stress effects on cortisol levels exhibited gender differences; 
the primary interaction was significant in men $(\mathrm{F}(2,50)=4.842, \mathrm{p}=.012)$ but not women $(\mathrm{F}(2,47)=0.898, \mathrm{p}=.414)$.

Statistics to test the efficacy of the stress manipulation were run on the full usable sample (N $\leq 54$ ); for all other analyses, only the sub-sample showing self-report ratings consistent with group assignment were included $(\mathrm{N}=47$; see section 2.6.1).

\subsection{Effects of Stress on Memory Performance}

3.2.1. Group Effects-Figure $2 \mathrm{~A}$ illustrates the effects of stress group and valence on dprime. There were no main effects of group or valence (p's > 0.05, Supplemental Table 1); however, there was a significant crossover interaction $(\mathrm{F}(1,45)=4.253, \mathrm{p}=.045)$ such that stress resulted in an enhancement of memory for neutral images and a decrement in memory for negative images. To elucidate the source of this interaction, we conducted similar ANOVAs separately using hit rate and false alarm rate as dependent variables.

There was a main effect of valence on hit rate such that negative images were more likely to be correctly remembered as old $(F(1,45)=45.330, p<.001$; Figure $2 B)$. However, there were no main or interacting effects with stress group (Supplemental Table 1). This pattern of results suggests that negative valence increased hit rate equally across stress and control groups.

There was an interaction of group and valence on false alarm rates such that there were more negative than neutral false alarms in the stress group but no such difference in the control group $(F(1,45)=9.165, p=.004$; Figure $2 C)$. This effect was notably consistent at the individual subject level, with only two participants in the stress group exhibiting more false alarms for neutral images (Figure 3). There was also a significant main effect of valence $(\mathrm{F}(1,45)=7.503, \mathrm{p}=.009)$ but not group $(\mathrm{F}(1,45)=0.327, \mathrm{p}=.57)$ on false alarm rates, such that negative valence resulted in more false alarms than neutral valence. Post-hoc analyses showed that self-reported confidence did not interact with group $(\mathrm{p}=.998)$, valence $(\mathrm{p}=.942)$, or group*valence $(\mathrm{p}=.567)$ to explain differences in false alarm rates (see Supplemental Table 3 for full model statistics). Together, these findings suggest that the difference in performance induced by stress was driven by differences in false recognition.

3.2.2. Individual Differences in Stress-To further investigate stress-related differences in false alarm rates, we assessed whether individual differences in engagement of the components of the stress response (self-report, cortisol, and heart rate) were related to false alarm rates for negative and neutral images. We found a significant interaction between self-reported stress and valence $(F(1,45)=14.901 ; p=.001$, FDR corrected $)$. Consistent with group-level results, the greater stress participants reported, the greater difference there was between their negative and neutral false alarm rates (Fig. 4A). A significant interaction between valence and math-induced heart rate exhibited the same pattern $(F(1,39)=7.355, p$ $=.015$, FDR corrected; Fig. 4B). There was no such interaction between valence and evoked cortisol levels $(F(1,44)=0.794, p=.378$, FDR corrected; Fig. 4C). 


\subsection{Exploratory Analyses on Source Memory Attribution for False Alarms}

To test how vividly participants falsely recalled new information, we tested whether they attributed a source to the false alarm memories (i.e., seeing it in the "look" or "decrease" condition versus selecting "I don't know"). Notably, any source attributions given after a false alarm are incorrect because participants never previously viewed these images during encoding. Participants cannot make correct source memory judgments here, they can only decide whether to make an incorrect source attribution. Thus, this analysis characterizes the vividness of false recollections rather than source memory accuracy. We found a trending crossover interaction between valence and group $(\mathrm{p}=0.082$; Supplemental Table 2), such that control participants were more likely to make a source attribution for neutral images than negative images, but stressed participants were more likely to do so for negative images than neutral images (Fig. 5). This pattern of results suggests that not only were stressed participants more likely to make false alarms for a negative image, these false recollections were also more vivid for negative compared to neutral images.

\section{Discussion}

In the present study, we characterized the effects of stress on emotional memory. By having participants anticipate giving a speech and repeatedly exposing them to a timed and socially evaluative math task, we reliably elicited a robust stress response during memory encoding, as indicated by self-reported stress as well as increases in cortisol and heart rate. We found that stress significantly influenced emotional memory, such that memory was better for neutral pictures and worse for negative pictures when encoded under stress. By decomposing memory retrieval into its constituent elements, we characterized how this interaction emerged. Whereas negative valence increased participants' ability to identify old images regardless of the stress condition, stress changed participants' susceptibility to endorsing competing new information as old. Stress reduced false alarms for neutral images, and enhanced false alarms for negative images. Individual difference analyses revealed that these effects were largest for participants who were most stressed. Furthermore, not only were stressed participants more likely to falsely remember a negative image that had never been previously seen, they were also more likely to claim this unseen negative image came from a specific source, suggesting a more vivid false recollection. Together, these data suggest that stress undermines the benefits of emotion on memory by changing individuals' susceptibility towards false alarms.

A large body of research has investigated the influences of stress on valence-specific memory effects. While effects have been highly variable across studies (Shields et al., 2017), some prior research has found that psychological stress enhances emotional memory encoding, either by improving long-term memory for emotional material or hindering it for neutral material (Payne et al., 2006, 2007), which contradicts the current findings. Critically, our research protocol differs from most prior work investigating interactions between stress and emotional memory. Prior research typically induces stress with a single stressful event, which has the potential to decay rapidly. The present study used a repeated stress elicitation procedure, which may have resulted in a more sustained, higher level of continuous stress. In other memory studies, the effects of stress on behavior have been shown to be non-linear, 
such that moderate and high levels of stress hormones result in divergent findings (Abercrombie et al., 2003; Domes, Rothfischer, Reichwald, \& Hautzinger, 2005; Schilling et al., 2013). Thus, the more sustained high levels of stress induced in the present study may have been sufficiently different from previous work in their total intensity to result in contrary effects on emotional memory. In line with this interpretation, previous research utilizing a repeated stress procedure to induce sustained stress also found enhanced memory for neutral pictures under stress (Henckens et al., 2009); albeit, this study found that sustained stress also enhanced memory for negative pictures. Future work is needed to systematically characterize how varying the duration and intensity of stressful events differentially influences memory for neutral and negative events.

To better understand how stress influenced memory encoding, we aimed to decompose measures of memory performance into constituent parts. There are at least two paths to improved memory performance: improving hit rates and reducing false alarm rates. Improving hit rates - correctly identifying previously seen material as old — represents strengthening the memory representation of an observed event. In the present study, participants exhibited greater hit rates for negative compared to neutral images. Critically, however, stress did not modulate this effect. Previous work has consistently shown improved hit rate for negative material, presumably due to stronger memory representations or greater "signal" (Hamann, 2001; Yonelinas \& Ritchey, 2015). Thus, we found that stress does not alter the ability of negative valence to increase the strength of memory representations during encoding, which raises the interesting possibility that stress and negative valence may increase hit rates by similar mechanisms.

The other path to better memory performance is to decrease false alarm rates- incorrectly endorsing never-before-seen material as old-which represents improving the fidelity of the memory representation, or making the representation more distinct. Regardless of how strong a memory representation is, if it is less distinct (i.e. lacking information about precise details), it will be more difficult to discriminate from other images that have similar properties, resulting in false alarms. Here, stress at encoding resulted in increased false alarms for negative images and decreased false alarms for neutral images at retrieval two weeks later. Thus, we found that stress alters the discriminability of stimuli, making neutral images more discriminable and negative images less discriminable.

Much of the prior work has focused on how stress influences hit rates or overall memory performance, with less attention being directed towards false alarms. Research outside of the domain of stress, however, has shown that encoding negative images is more likely to result in greater false alarms and less distinct memories than encoding neutral images (BessetteSymons, 2018; Dougal \& Rotello, 2007; Rimmele, Davachi, Petrov, Dougal, \& Phelps, 2011). We believe that the same processes increasing false alarms to negative images may be exacerbated under stress. Specifically, the decrease in encoding of memories that are distinctive for negative images is likely subserved by increases in amygdala activation during emotional compared to neutral stimuli. Increase activation of the amygdala has been shown to shunt memory encoding from the hippocampus, which typically produces rich detailed memory traces (reviewed by Murty \& Adcock, 2017; Yonelinas \& Ritchey, 2015), to the perirhinal cortex, which typically produces more gestalt or indistinct memories 
(Eichenbaum, Yonelinas, \& Ranganath, 2007; Murty \& Adcock, 2017; Wang, Ranganath, \& Yonelinas, 2014). Meanwhile, stress and its neurobiological correlates strengthen amygdala functioning in response to aversive stimuli (Arnsten, 2009; Taylor et al., 2008; van Stegeren et al., 2007), which could magnify amygdala-mediated increases in low-fidelity memory for negative pictures, resulting in greater false alarms for negative pictures under stress, as is seen in the present study.

While this putative mechanism can explain increases in false alarm rates for negative images, it does not explain decreases in false alarm rates for neutral images. However, recent models of neuromodulation suggests that arousal-inducing factors like stress can bias competition of neural processing towards the most salient stimuli, while decreasing processing away from less salient stimuli (Mather, Clewett, Sakaki, \& Harley, 2016). We predict a parallel mechanism may occur during memory encoding, such that under states of stress, amygdala neuromodulation is prioritized towards negative images resulting in less hippocampal-dependent encoding, and away from neutral images resulting in more hippocampal-dependent encoding. This putative mechanism would in turn protect neutral stimuli more from arousal-based disruptions in memory fidelity in stressed versus nonstressed states. Recently, stress has been shown to facilitate hippocampal-dependent memory encoding — which is thought to mediate high fidelity memory representations - for neutral images when they were presented intermixed with negative images (Ritchey, McCullough, Ranganath, \& Yonelinas, 2017). Meanwhile, stress has been shown to decrease hippocampal-dependent memory encoding and increase false alarm rates for neutral images presented in isolation (Qin, Hermans, Marle, \& Fernández, 2012). Together these findings suggest that stress may negatively impact the fidelity of the most salient stimuli presented during encoding, but enhance memory fidelity for relatively less salient stimuli.

The current study presents preliminary evidence that stress negatively impacts emotional memory encoding by decreasing the fidelity of negative stimuli in long-term memory. However, further studies are needed to characterize the mechanisms and boundary conditions for this effect. While our current results are in line with prior research detailing arousal's influence on memory encoding (Murty \& Adcock, 2017), studies implementing neuroimaging and psychophysiological approaches in humans, as well as parallel studies in animal models, are needed to support our proposed mechanism. The current study also only tested memory at a delay, which did not allow us to characterize how memories may have changed over time. This factor may be especially important, as previous research has emphasized critical roles for sleep and memory consolidation in mediating stress and emotion's influence on memory encoding (McGaugh, 2004; Payne \& Kensinger, 2010). Another critical area of research is to determine if the characterized stress effects differ across sexes, as arousal's modulatory role on episodic memory formation is known to interact with sex hormones (Cahill, 2010; Herrera, Wang, \& Mather, 2018). However, the present study did not control for factors such as birth control use and menstrual phase, and thus was not designed to assess these differences. Another important caveat to the present conclusions is that participants also viewed trials for which they had to regulate their emotional response, which were not analyzed in the current report but may have influenced how stimuli in other conditions were viewed. However, previous work on the same data found that stress did not impact reappraisal-related processing (Shermohammed et al., 
2017). Furthermore, valence effects in the control condition were similar to previous work that did not include reappraisal trials (LaBar \& Cabeza, 2006). It is therefore unlikely that the presence of reappraisal trials contaminated the stress-valence memory interactions observed here. Finally, the current study investigated stress as a single state occurring over the entire experiment. However, stress responses are dynamic (Joëls, Fernandez, \& Roozendaal, 2011); different stages of the response may interact with emotional memory in different ways. Future analyses are needed to assess individual differences in dynamic changes in emotional responding and stress reactivity over time, as these studies may provide a more nuanced characterization of stress as a sequence of events that unfold over time.

In the present study, acute stress modulated memory distinctiveness without impacting memory strength. Stressed participants exhibited more false alarms to negative images, indicative of less distinct memory representations, and fewer false alarms for neutral images. Strikingly, our data show that a context manipulation at encoding can affect the response to new information that had never even been seen, and suggest that the influence of stress can have long-lasting influences on subsequent retrieval-related processes. Meanwhile, emotional content similarly enhanced hit rates in stress and control groups, suggesting no influence of stress on the strength of memory signals. The combination of these components resulted in stress-mediated impairments in memory performance for negative material and improvements for neutral material. The unintuitive nature of this result suggests a novel synthesis of previous mechanistic work on the interactions between stress and valence with the amygdala and its targets in the medial temporal lobe (i.e., hippocampus versus perirhinal cortex). Furthermore, these findings demonstrate that the influence of stress on memory encoding is both content-dependent and nuanced. In order to appreciate this complexity, it is critical to consider memory distinctiveness in addition to memory strength. For example, the stressed witness to the crime introduced at the beginning of this article may indeed have a strong recollection of the criminal, but may also have an impoverished ability to discriminate the assailant from other individuals in a police lineup. In order to understand what is truly driving changes in memory, it is essential that future researchers consider and report false alarms as well as hits when assessing the influence of moderating factors on memory performance.

\section{Supplementary Material}

Refer to Web version on PubMed Central for supplementary material.

\section{Acknowledgements}

We thank Pranjal H. Mehta and Cassandra M. Brandes for guidance on the cortisol collection protocol and Joan Zhang for helpful discussion. Research reported in this publication was supported through a contract with the US Army Natick Soldier Research, Development, and Engineering Center (Natick, MA) under award number W911QY-14-C-0009 to L.H.S., by the National Science Foundation (DGE1144152 to M.S.), and by the National Institutes of Health Shared Information Grant (S10OD020039). 


\section{References}

Abercrombie HC, Kalin NH, Thurow ME, Rosenkranz MA, \& Davidson RJ (2003). Cortisol variation in humans affects memory for emotionally laden and neutral information. Behavioral Neuroscience, 117(3), 505-516. 10.1037/0735-7044.117.3.505 [PubMed: 12802879]

Anisman H, \& Merali Z (1999). Understanding stress: characteristics and caveats. Alcohol Research \& Health: The Journal of the National Institute on Alcohol Abuse and Alcoholism, 23(4), 241-249. [PubMed: 10890820]

Arnsten AFT (2009). Stress signalling pathways that impair prefrontal cortex structure and function. Nature Reviews. Neuroscience, 10(6), 410-422. 10.1038/nrn2648 [PubMed: 19455173]

Bessette-Symons BA (2018). The robustness of false memory for emotional pictures. Memory, 26(2), 171-188. 10.1080/09658211.2017.1339091 [PubMed: 28625103]

Buchanan TW, \& Lovallo WR (2001). Enhanced memory for emotional material following stress-level cortisol treatment in humans. Psychoneuroendocrinology, 26(3), 307-317. 10.1016/ S0306-4530(00)00058-5 [PubMed: 11166493]

Cahill L (2010). Sex influences on brain and emotional memory: the burden of proof has shifted. Progress in Brain Research, 186, 29-40. 10.1016/B978-0-444-53630-3.00003-8 [PubMed: 21094884]

Cahill L, \& McGaugh JL (1998). Mechanisms of emotional arousal and lasting declarative memory. Trends in Neurosciences, 21(7), 294-299. 10.1016/S0166-2236(97)01214-9 [PubMed: 9683321]

Cahill L, Prins B, Weber M, \& McGaugh JL (1994). $\beta$-Adrenergic activation and memory for emotional events. Nature, 371(6499), 702-704. 10.1038/371702a0 [PubMed: 7935815]

Cornelisse S, van Stegeren AH, \& Joëls M (2011). Implications of psychosocial stress on memory formation in a typical male versus female student sample. Psychoneuroendocrinology, 36(4), 569578. 10.1016/j.psyneuen.2010.09.002 [PubMed: 20933337]

de Quervain DJ-F, Roozendaal B, Nitsch RM, McGaugh JL, \& Hock C (2000). Acute cortisone administration impairs retrieval of long-term declarative memory in humans. Nature Neuroscience, 3(4), 313-314. 10.1038/73873 [PubMed: 10725918]

Dedovic K, Renwick R, Mahani NK, Engert V, Lupien SJ, \& Pruessner JC (2005). The Montreal Imaging Stress Task: Using functional imaging to investigate the effects of perceiving and processing psychosocial stress in the human brain. In Journal of Psychiatry and Neuroscience (Vol. 30, pp. 319-325). [PubMed: 16151536]

Domes G, Rothfischer J, Reichwald U, \& Hautzinger M (2005). Inverted-U Function Between Salivary Cortisol and Retrieval of Verbal Memory After Hydrocortisone Treatment. Behavioral Neuroscience, 119(2), 512-517. 10.1037/0735-7044.119.2.512 [PubMed: 15839797]

Dougal S, \& Rotello CM (2007). "Remembering" emotional words is based on response bias, not recollection. Psychonomic Bulletin \& Review, 14(3), 423-429. 10.3758/BF03194083 [PubMed: 17874582]

Eichenbaum H, Yonelinas AP, \& Ranganath C (2007). The medial temporal lobe and recognition memory. Annual Review of Neuroscience, 30, 123-152. 10.1146/annurev.neuro. 30.051606 .094328

Hamann S (2001). Cognitive and neural mechanisms of emotional memory. Trends in Cognitive Sciences, 5(9), 394-400. 10.1016/S1364-6613(00)01707-1 [PubMed: 11520704]

Hellhammer DH, Wüst S, \& Kudielka BM (2009). Salivary cortisol as a biomarker in stress research. Psychoneuroendocrinology, 34(2), 163-171. 10.1016/j.psyneuen.2008.10.026 [PubMed: 19095358]

Henckens MJAG, Hermans EJ, Pu Z, Joëls M, \& Fernández G (2009). Stressed memories: how acute stress affects memory formation in humans. The Journal of Neuroscience : The Official Journal of the Society for Neuroscience, 29(32), 10111-10119. 10.1523/JNEUROSCI.1184-09.2009 [PubMed: 19675245]

Hoscheidt SM, LaBar KS, Ryan L, Jacobs WJ, \& Nadel L (2014). Encoding negative events under stress: High subjective arousal is related to accurate emotional memory despite misinformation exposure. Neurobiology of Learning and Memory, 112, 237-247. 10.1016/J.NLM.2013.09.008 [PubMed: 24055594] 
Jelici M, Geraerts E, Merckelbach H, \& Guerrieri R (2004). Acute stress enhances memory for emotional words, but impairs memory for neutral words. The International Journal of Neuroscience, 114(10), 1343-1351. 10.1080/00207450490476101 [PubMed: 15370191]

Joëls M, Fernandez G, \& Roozendaal B (2011). Stress and emotional memory: a matter of timing. Trends in Cognitive Sciences, 15(6), 280-288. 10.1016/j.tics.2011.04.004 [PubMed: 21571575]

Kirschbaum C, Pirke KM, \& Hellhammer DH (1993). The 'Trier Social Stress Test'--a tool for investigating psychobiological stress responses in a laboratory setting. Neuropsychobiology, 28(12), 76-81. https://doi.org/119004 [PubMed: 8255414]

LaBar KS, \& Cabeza R (2006). Cognitive neuroscience of emotional memory. Nature Reviews Neuroscience, 7(1), 54-64. 10.1038/nrn1825 [PubMed: 16371950]

Lang PJ, Bradley MM, \& Cuthbert BN (1997). International Affective Picture System (IAPS): Technical Manual and Affective Ratings. NIMH Center for the Study of Emotion and Attention, 39-58. 10.1016/j.epsr.2006.03.016

Macmillan NA, \& Creelman CD (2005). Detection Theory: A User's Guide. Detection theory: A user's guide (2nd ed.). (2nd ed.). Lawrence Elbaum Associates, Inc 10.1017/ CBO9781107415324.004

Maheu FS, Collicutt P, Kornik R, Moszkowski R, \& Lupien SJ (2005). The perfect time to be stressed: A differential modulation of human memory by stress applied in the morning or in the afternoon. Progress in Neuro-Psychopharmacology and Biological Psychiatry, 29(8), 1281-1288. 10.1016/ J.PNPBP.2005.08.012 [PubMed: 16229931]

Mather M, Clewett D, Sakaki M, \& Harley CW (2016). Norepinephrine ignites local hotspots of neuronal excitation: How arousal amplifies selectivity in perception and memory. The Behavioral and Brain Sciences, 39, e200 10.1017/S0140525X15000667 [PubMed: 26126507]

McGaugh JL (2004). The Amygdala Modulates the Consolidation of Memories of Emotionally Arousing Experiences. Annual Review of Neuroscience, 27(1), 1-28. 10.1146/annurev.neuro. 27.070203.144157

Murty VP, \& Adcock RA (2017). Distinct Medial Temporal Lobe Network States as Neural Contexts for Motivated Memory Formation. The Hippocampus from Cells to Systems (pp. 467-501). Cham: Springer International Publishing 10.1007/978-3-319-50406-3_15

Payne JD, Jackson ED, Hoscheidt S, Ryan L, Jacobs WJ, \& Nadel L (2007). Stress administered prior to encoding impairs neutral but enhances emotional long-term episodic memories. Learning \& Memory, 14(12), 861-868. 10.1101/lm.743507 [PubMed: 18086830]

Payne JD, Jackson E, Ryan L, Hoscheidt S, Jacobs J, \& Nadel L (2006). The impact of stress on neutral and emotional aspects of episodic memory. Memory, 14(1), 1-16. 10.1080/09658210500139176 [PubMed: 16423737]

Payne JD, \& Kensinger EA (2010). Sleep's Role in the Consolidation of Emotional Episodic Memories. Current Directions in Psychological Science, 19(5), 290-295. $10.1177 / 0963721410383978$

Pruessner JC, Kirschbaum C, Meinlschmid G, \& Hellhammer DH (2003). Two formulas for computation of the area under the curve represent measures of total hormone concentration versus time-dependent change. Psychoneuroendocrinology, 28(28), 916-931. 10.1016/ S0306-4530(02)00108-7 [PubMed: 12892658]

Qin S, Hermans EJ, van Marle HJF, \& Fernández G (2012). Understanding Low Reliability of Memories for Neutral Information Encoded under Stress: Alterations in Memory-Related Activation in the Hippocampus and Midbrain. Journal of Neuroscience, 32(12), 4032-4041. 10.1523/JNEUROSCI.3101-11.2012 [PubMed: 22442069]

Rimmele U, Davachi L, Petrov R, Dougal S, \& Phelps EA (2011). Emotion enhances the subjective feeling of remembering, despite lower accuracy for contextual details. Emotion, 11(3), 553-562. 10.1037/a0024246 [PubMed: 21668106]

Rimmele U, Lackovic SF, Tobe RH, Leventhal BL, \& Phelps EA (2016). Beta-adrenergic Blockade at Memory Encoding, but Not Retrieval, Decreases the Subjective Sense of Recollection. Journal of Cognitive Neuroscience, 28(6), 895-907. 10.1162/jocn_a_00941 [PubMed: 26942318] 
Ritchey M, McCullough AM, Ranganath C, \& Yonelinas AP (2017). Stress as a mnemonic filter: Interactions between medial temporal lobe encoding processes and post-encoding stress. Hippocampus, 27(1), 77-88. 10.1002/hipo.22674 [PubMed: 27774683]

Rotello CM, \& Macmillan NA (2007). Response Bias in Recognition Memory. Psychology of Learning and Motivation, 48, 61-94. 10.1016/S0079-7421(07)48002-1

Schilling TM, Kölsch M, Larra MF, Zech CM, Blumenthal TD, Frings C, \& Schächinger H (2013). For whom the bell (curve) tolls: cortisol rapidly affects memory retrieval by an inverted U-shaped dose-response relationship. Psychoneuroendocrinology, 38(9), 1565-1572. 10.1016/j.psyneuen. 2013.01.001 [PubMed: 23374327]

Schwabe L, \& Wolf OT (2010). Learning under stress impairs memory formation. Neurobiology of Learning and Memory, 93(2), 183-188. 10.1016/J.NLM.2009.09.009 [PubMed: 19796703]

Shermohammed M, Mehta PH, Zhang J, Brandes CM, Chang LJ, \& Somerville LH (2017). Does Psychosocial Stress Impact Cognitive Reappraisal? Behavioral and Neural Evidence. Journal of Cognitive Neuroscience, 29(11), 1803-1816. 10.1162/jocn [PubMed: 28598734]

Shields GS, Sazma MA, McCullough AM, \& Yonelinas AP (2017). The Effects of Acute Stress on Episodic Memory: A Meta-Analysis and Integrative Review. Psychological Bulletin 10.1037/ bu10000100

Smeets T, Jelicic M, \& Merckelbach H (2006). The effect of acute stress on memory depends on word valence. International Journal of Psychophysiology, 62(1), 30-37. 10.1016/J.IJPSYCHO. 2005.11.007 [PubMed: 16388863]

Smeets T, Otgaar H, Candel I, \& Wolf OT (2008). True or false? Memory is differentially affected by stress-induced cortisol elevations and sympathetic activity at consolidation and retrieval. Psychoneuroendocrinology, 33(10), 1378-1386. 10.1016/j.psyneuen.2008.07.009 [PubMed: 18790572]

Taylor SE, Burklund LJ, Eisenberger NI, Lehman BJ, Hilmert CJ, \& Lieberman MD (2008). Neural bases of moderation of cortisol stress responses by psychosocial resources. Journal of Personality and Social Psychology, 95(1), 197-211. 10.1037/0022-3514.95.1.197 [PubMed: 18605860]

van Stegeren AH, Wolf OT, Everaerd W, Scheltens P, Barkhof F, \& Rombouts SARB (2007). Endogenous cortisol level interacts with noradrenergic activation in the human amygdala. Neurobiology of Learning and Memory, 87(1), 57-66. 10.1016/j.nlm.2006.05.008 [PubMed: 16884932]

Wang W-C, Ranganath C, \& Yonelinas AP (2014). Activity reductions in perirhinal cortex predict conceptual priming and familiarity-based recognition. Neuropsychologia, 52, 19-26. 10.1016/ j.neuropsychologia.2013.10.006 [PubMed: 24157537]

Ycaza Herrera A, Wang J, \& Mather M (2018). The gist and details of sex differences in cognition and the brain: How parallels in sex differences across domains are shaped by the locus coeruleus and catecholamine systems. Progress in Neurobiology 10.1016/j.pneurobio.2018.05.005

Yonelinas AP, \& Ritchey M (2015). The slow forgetting of emotional episodic memories: an emotional binding account. Trends in Cognitive Sciences, 19(5), 259-267. 10.1016/J.TICS.2015.02.009

[PubMed: 25836045] 


\section{Highlights}

- $\quad$ Stress impairs overall memory for negative images, but improves it for neutral images

- $\quad$ Effects driven by false alarms, which increase for negative images under stress

- $\quad$ Effects were largest for individuals who were most stressed

- $\quad$ Stress did not affect ability to identify previously seen images (memory "hits") 


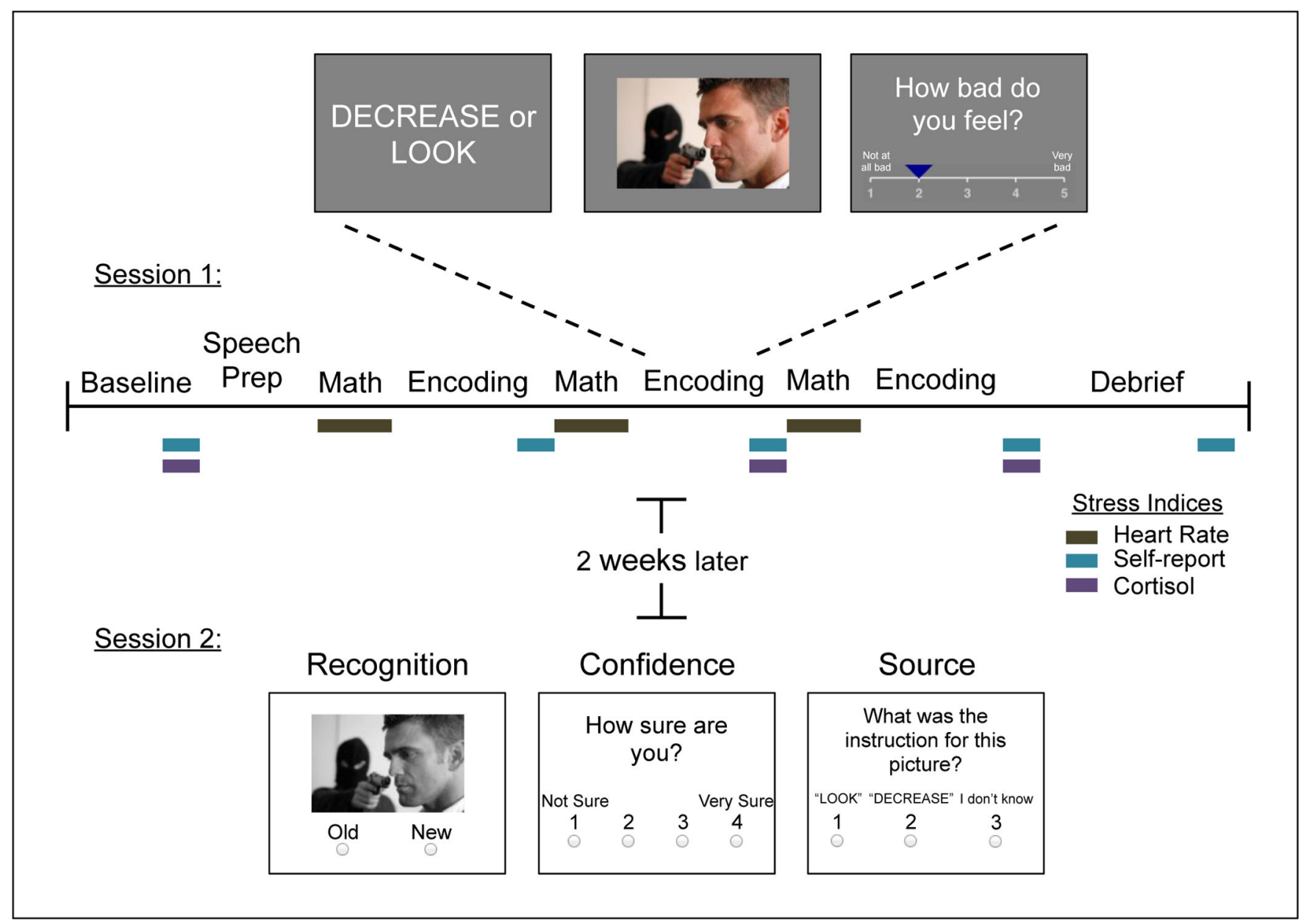

Figure 1.

Study design. The upper timeline describes the first session, when the stress induction and memory encoding occurred. Three indices of stress were acquired during this visit, denoted by colored boxes. Memory retrieval occurred approximately 2 weeks after encoding in Session 1. 


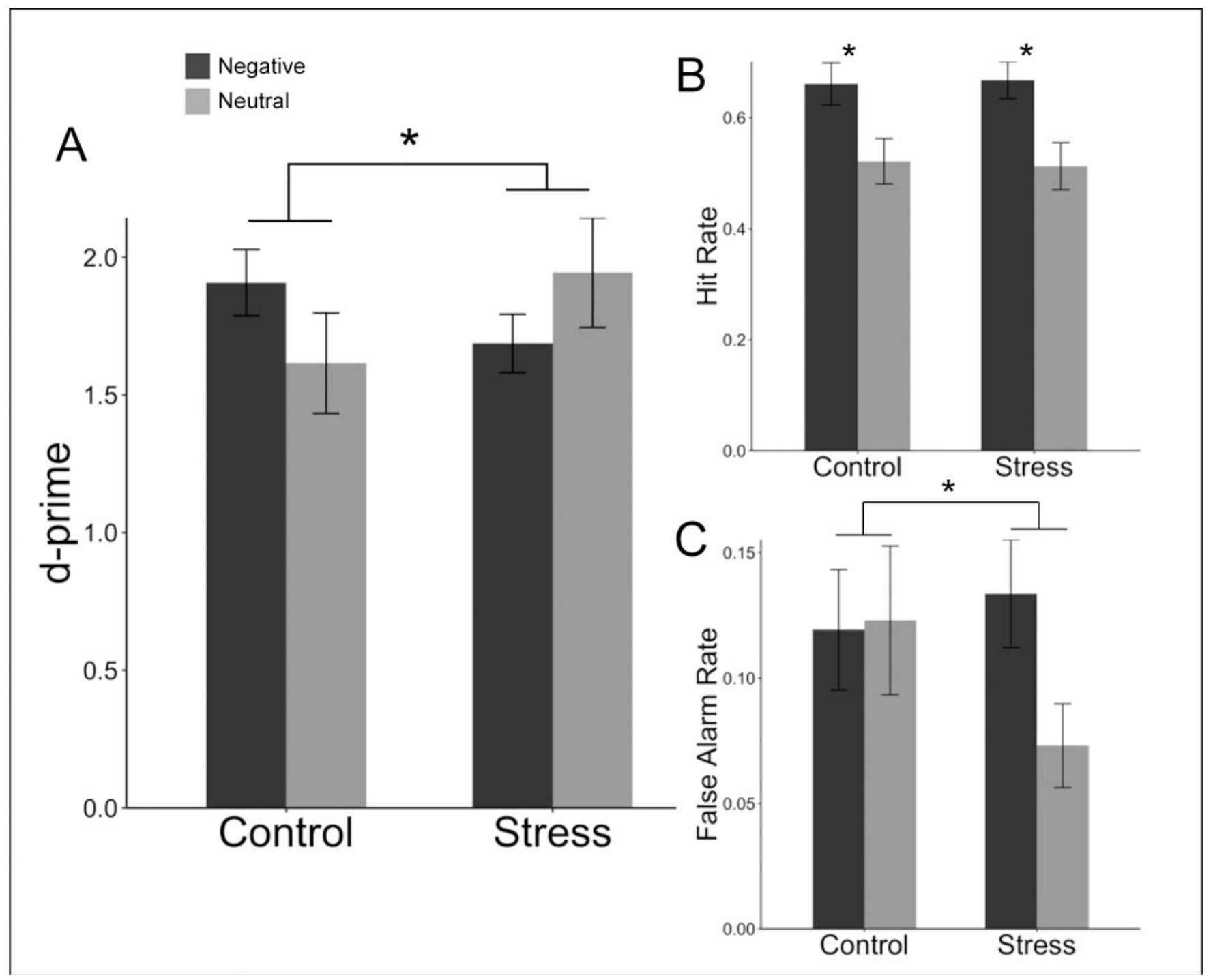

Figure 2.

Group differences in (A) d-prime, (B) hit rate, and (C) false alarm rate for negative and neutral pictures. There was a significant main effect of valence for hit rate, and a significant interaction between valence and group for d-prime and false alarm rate. Error bars are SEM. 


\section{Individual Differences in Negative - Neutral FAs}

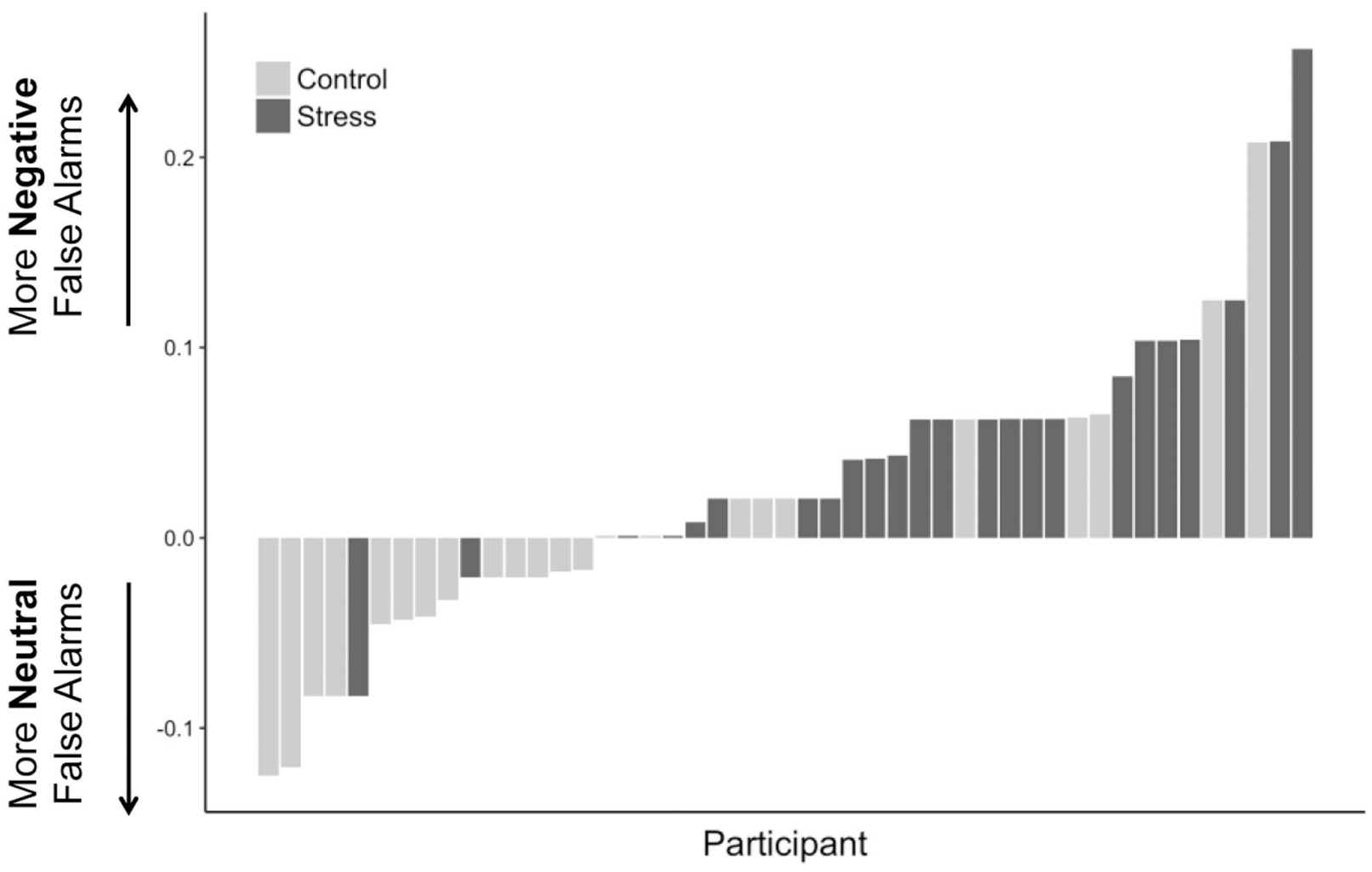

Figure 3.

Plotted is each participant's false alarm rate for negative minus neutral pictures. Values above zero indicate more negative than neutral false alarms and values below zero indicate more neutral than negative false alarms. Shading indicates group assignment. Generally, participants in the stress group (black) exhibited more false alarms for negative pictures and participants in the control group (gray) exhibited more false alarms for neutral pictures. 


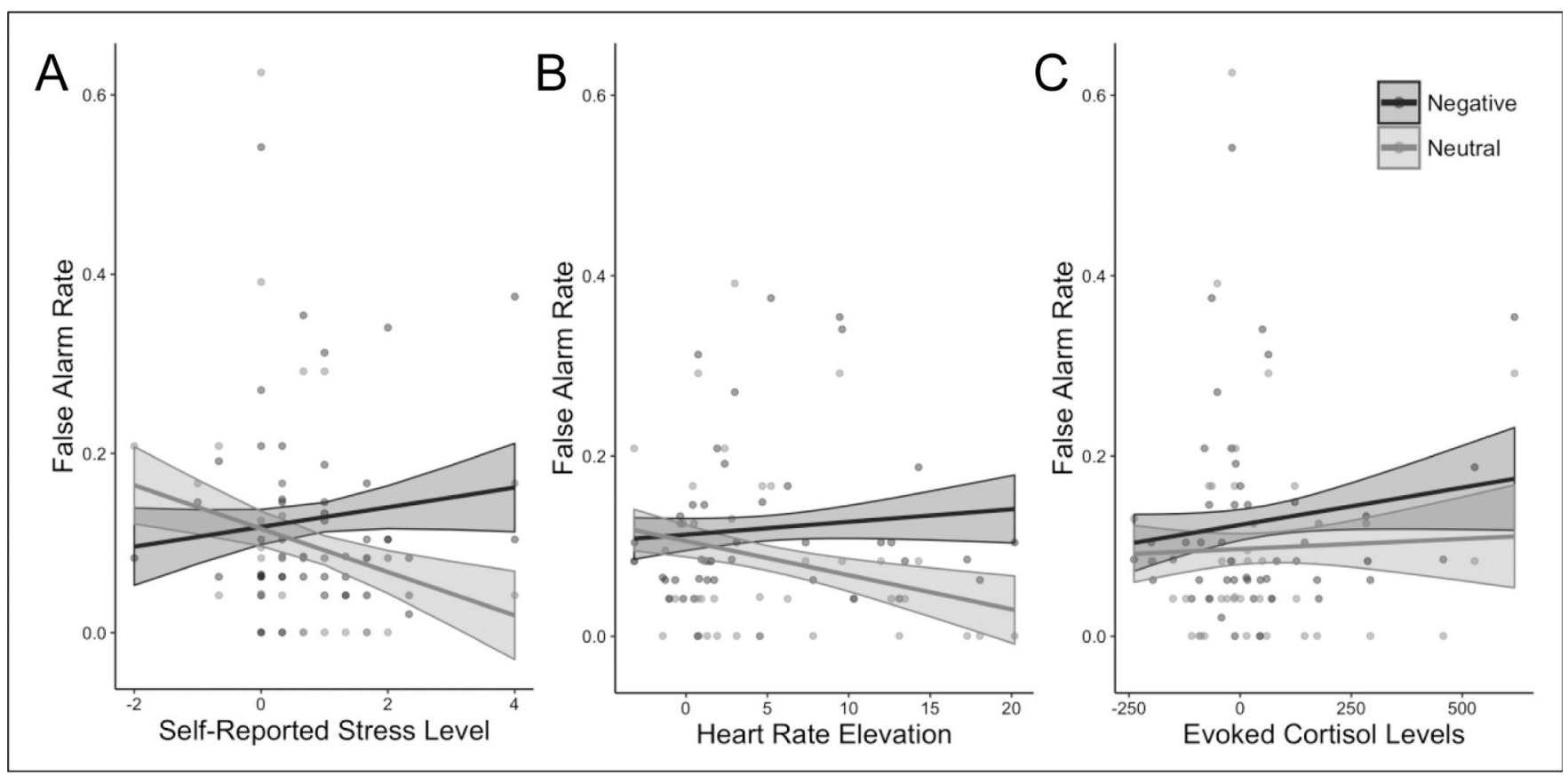

\section{Figure 4.}

Individual differences in stress and false alarms. Irrespective of group assignment, increases in evoked stress as assessed by A) self-report $(\mathrm{N}=47 ; \mathrm{F}(1,45)=14.901 ; \mathrm{p}=.001)$ and $\mathrm{B})$ heart rate $(\mathrm{N}=41 ; \mathrm{F}(1,39)=7.355, \mathrm{p}=.015)$ correlated with greater divergence in false alarm rates between negative and neutral images. C) No such correlation was evident with cortisol levels $(\mathrm{N}=46 ; \mathrm{F}(1,44)=0.794, \mathrm{p}=.378)$. Shaded areas correspond to the standard error of fitted values. 


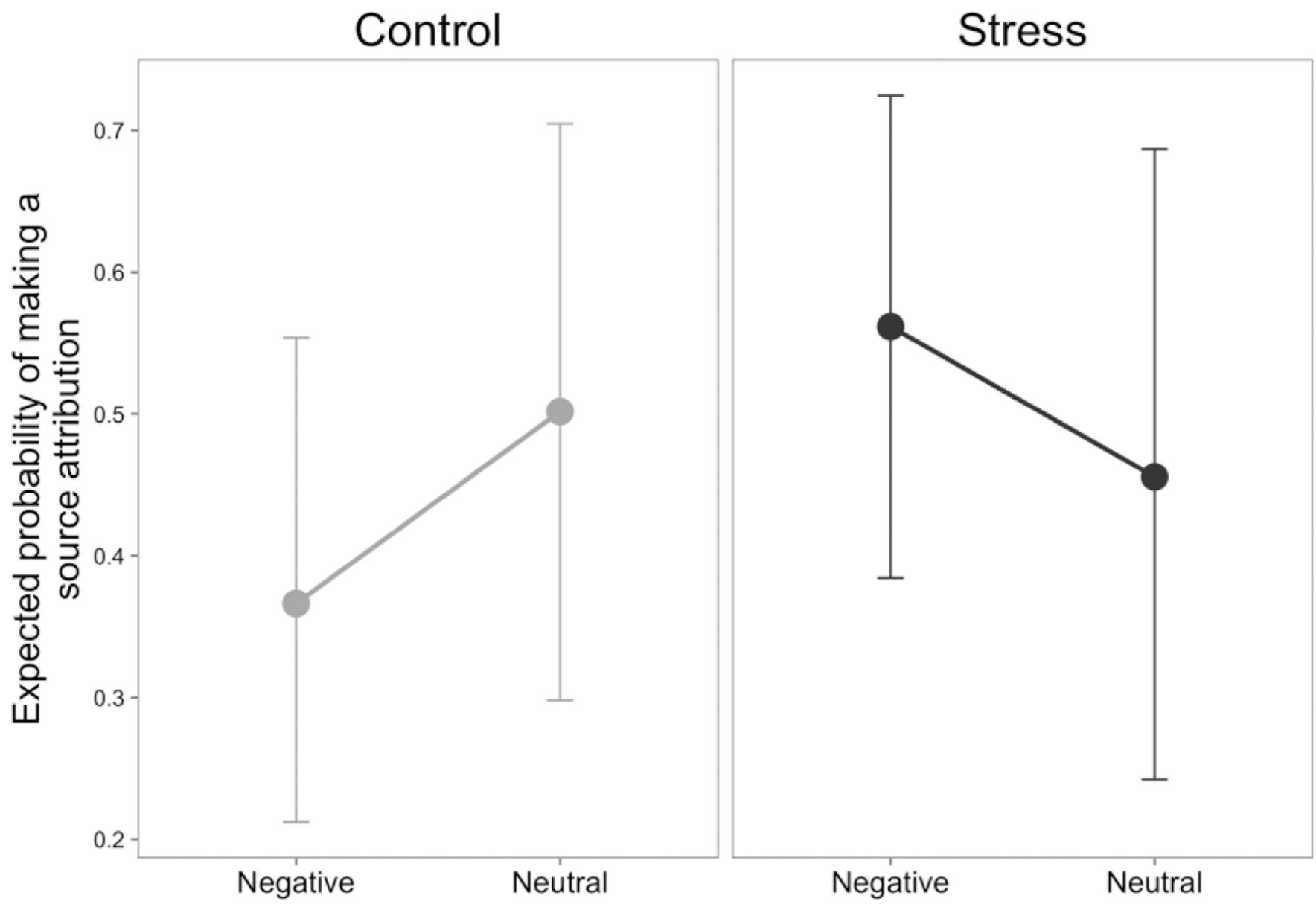

Figure 5.

False alarm source attribution. Source attribution data (whether participants chose to try to identify if the retrieved image came from a "LOOK" or "DECREASE" trial, versus saying they did not know) for false alarm trials was subject to a mixed-effects logistic regression. Plotted are the probabilities predicted from the model of making a source attribution in each condition. A trending ( $\mathrm{p}=0.082)$ interaction between stress and valence suggested that stressed participants (black) had more vivid false recollections for negative material, while control participants (gray) had more vivid false recollections for neutral material. 